\section{BMJ Open} Ophthalmology

\title{
Prediction of myopia onset with refractive error measured using non- cycloplegic subjective refraction: the WEPrOM Study
}

\author{
Yee Ling Wong (1) ," Yimin Yuan, ${ }^{2,3}$ Binbin Su (D) ,,3 Shezad Tufail, ${ }^{1}$ \\ Yang Ding (D) , ${ }^{2,3}$ Yingying Ye, ${ }^{2}$ Damien Paille, ${ }^{1}$ Björn Drobe, ${ }^{1,3}$ Hao Chen (D) , \\ Jinhua $\mathrm{Bao}^{2,3}$
}

To cite: Wong YL, Yuan Y, Su B, et al. Prediction of myopia onset with refractive error measured using non-cycloplegic subjective refraction: the WEPrOM Study. BMJ Open Ophthalmology 2021;6:e000628. doi:10.1136/ bmjophth-2020-000628

YLW and YY contributed equally.

Received 29 December 2020 Accepted 19 May 2021
Check for updates

\section{(c) Author(s) (or their} employer(s)) 2021. Re-use permitted under CC BY-NC. No commercial re-use. See rights and permissions. Published by BMJ.

\section{${ }^{1}$ R\&D AMERA, Essilor} International, Singapore ${ }^{2}$ Eye Hospital and School of Ophthalmology and Optometry, Wenzhou Medical University, Wenzhou, Zhejiang, China ${ }^{3}$ Wenzhou Medical UniversityEssilor International Research Centre (WEIRC), Wenzhou Medical University, Wenzhou, Zhejiang, China

\section{Correspondence to} Dr Jinhua Bao; baojessie@163. com

\section{ABSTRACT}

Background/aims To evaluate the predictive performance of various predictors, including noncycloplegic refractive error, for risk of myopia onset under pragmatic settings.

Methods The Wenzhou Medical University Essilor Progression and Onset of Myopia Study is a prospective cohort study of schoolchildren aged 6-10 years from two elementary schools in Wenzhou, China. Non-cycloplegic refraction, ocular biometry and accommodation measurements were performed. Myopia was defined as spherical equivalent (SE) $\leq-0.5$ diopter (D). ORs using multivariable logistic regression were determined. Area under the curve (AUC) evaluation for predictors was performed.

Results Schoolchildren who attended both baseline and 2-year follow-up were analysed ( $\mathrm{N}=1022)$. Of 830 non-myopic children at baseline, the 2-year incidence of myopia was $27.6 \%(95 \% \mathrm{Cl}, 24.2 \%$ to $31.3 \%)$. Female gender ( $\mathrm{OR}=2.2)$, more advanced study grades $(\mathrm{OR}=1.5)$, less hyperopic $S E(O R=11.5$ per $D)$, longer axial length $(A L$; $\mathrm{OR}=2.3$ per $\mathrm{mm})$, worse presenting visual acuity $(\mathrm{OR}=2.3$ per decimal), longer near work time $(\mathrm{OR}=1.1$ per hour/day) and lower magnitude of positive relative accommodation (PRA; OR=1.4 per D) were associated with myopia onset. PRA (AUC=0.66), SE (AUC=0.64) and $A L(A U C=0.62)$ had the highest AUC values. The combination of age, gender, parental myopia, SE, AL and PRA achieved an AUC of 0.74 . Conclusion Approximately one in four schoolchildren had myopia onset over a 2-year period. The predictors of myopia onset include lower magnitude of PRA, less hyperopic SE, longer AL and female gender. Of these, non-cycloplegic SE and PRA were the top single predictors, which can facilitate risk profiling for myopia onset.

\section{INTRODUCTION}

Myopia is a major public health issue. ${ }^{12}$ An earlier onset of myopia in childhood is linked to high myopia development. ${ }^{3}$ Complications of high degrees of myopia include myopic macular degeneration, glaucoma and retinal detachment, which could lead to visual impairment in later life. ${ }^{45}$ Numerous studies have

\section{Key messages}

What is already known about this subject?

Cycloplegic refractive error has high predictive performance for risk of myopia onset among children.

\section{What are the new findings?}

- Approximately one in four schoolchildren had myopia onset over a 2-year period.

$\checkmark$ Non-cycloplegic refractive error and accommodation ability had high predictive performance for risk of myopia onset, among other single factors including longer axial length and female gender that were associated with myopia onset among Chinese schoolchildren.

\section{How might these results change the focus of} research or clinical practice?

$>$ Non-cycloplegic spherical equivalent and positive relative accommodation can facilitate risk profiling of children at risk of myopia onset under pragmatic settings.

reported the prevalence of myopia, ranging from $4 \%$ to $48 \%$ among children worldwide. ${ }^{6}$ Specifically, the pooled prevalence of myopia among children aged 7-12 years in China was estimated at $30.7 \%$.

Numerous cohort studies in the USA, Australia, Singapore, Taiwan and China have reported the longitudinal changes in refraction, and the annualised incidence of myopia ranges from 3.4\%-33.6\% in Asian children ${ }^{8-15}$ to $2.2 \%-2.4 \%$ in Caucasian children. ${ }^{1617}$ Among primary schoolchildren, the annualised incidence of myopia was between $7.8 \%$ and $33.6 \%,{ }^{89111215}$ but the majority of these studies only had short follow-up periods of up to 1 year. There is a lack of longitudinal studies in China examining the incidence of myopia in young children with longer follow-up periods of $\geq 2$ years. ${ }^{9} 1115$ 
The predictors for myopia onset include parental myopia, ${ }^{11} 13141618$ female gender, ${ }^{9} 10121519$ longer axial length (AL), ${ }^{7818}$ reduced outdoor time, ${ }^{13} 1820$ increased near work time ${ }^{13}$ and accommodative insufficiency. ${ }^{21-24}$ Several studies have examined the contribution of the predictors to myopia onset. ${ }^{11} 161825-27$ Ocular parameters, such as baseline refractive error and ocular biometry, were the predictors with the largest contributions to predicting myopia onset. ${ }^{1126-28}$ To obtain spherical equivalent (SE) values, cycloplegia was used in most of these studies. However, cycloplegia is not readily available for use in most primary eye-care service providers. This limits the applicability of the use of cycloplegic SE as a predictor of myopia onset in a population setting, thus the assessment of the relevancy of SE obtained using noncycloplegic methods for myopia onset is important for practical insights. Given the side effects associated with the use of cycloplegic eye drops, there would be added value in evaluating the predictors for myopia onset based on non-cycloplegic outcomes. ${ }^{29}$ In combination with other predictors for myopia onset, predictive models using data that are readily accessible can aid prospective risk profiling and identification of children who are at higher risk of myopia onset for timely myopia control interventions. ${ }^{30}$

This study aims to examine the incidence of myopia in a school-based cohort study among Chinese schoolchildren in Wenzhou, China, and to evaluate the predictive performance of various predictors, including non-cycloplegic SE, ocular biometry and accommodation-related measurements, for risk of myopia onset.

\section{METHODS}

\section{Study design and population}

The Wenzhou Medical University Essilor Progression and Onset of Myopia (WEPrOM) Study was a prospective school-based cohort study conducted in Wenzhou of the Zhejiang province, China. The baseline examination was conducted in December 2014 and subsequent follow-up visits were conducted annually, for a total follow-up period of 3 years. The study included two schools in Wenzhou, one located in the urban city (PuXieShi campuses) and the other in the rural region (OuBei campus). All 1118 schoolchildren studying in elementary school grades 2 and 3 from the 2 schools were invited to participate in the WEPrOM Study, of which $1103(98.7 \%)$ attended the baseline examination. Of 1103 participants at baseline, $1090(98.8 \%), 1066(96.6 \%)$ and $945(85.7 \%)$ attended the 1-year, 2-year, and 3-year follow-up (loss to follow-up was mainly due to school transfers), respectively. Patients or the public were not involved in the design, or conduct, or reporting, or dissemination plans of our research. The study was conducted in accordance with the tenets of the Declaration of Helsinki. Informed written consent was obtained from the parents or guardians of all children.

\section{Inclusion criteria}

To examine the incidence of myopia and its associated predictors over a sufficiently long follow-up period with high participation rates, participants who attended both baseline and 2-year follow-up examinations with complete refraction data were included in this study.

\section{Visual acuity (VA) assessment, refractive error and ocular} biometry measurements

The following procedures were conducted by trained investigators, including ophthalmologists and optometrists, from The Eye Hospital of Wenzhou Medical University. The same procedures were conducted for each visit. The monocular presenting VA (PVA) was measured using the Standard Logarithmic Visual Acuity $\mathrm{E}$ Chart at $5 \mathrm{~m}$, with schoolchildren wearing their habitual correction (if any). Subjective refraction was performed based on retinoscopy on the same day by a trained ophthalmologist, with fogging lenses (working distance lenses of +2.00 D in the trial frame after retinoscopy). VA value was recorded as the one corresponding to the lowest line at which at least half of the optotypes were correctly identified (represented in decimals). Refractive data were presented in spherocylinder form, Sphere/ Cylinder x Axis, and transformed using Long's method ${ }^{31}$ for descriptive analyses. SE of refractive error was defined as sphere plus half cylinder. Myopia was defined as SE $\leq-0.5 \mathrm{D}$ in at least one eye. ${ }^{32} \mathrm{AL}$ was determined using Lenstar LS900 (Haag-Streit Koeniz, Switzerland) as the average of 3 recordings.

\section{Accommodation measurements}

Positive relative accommodation (PRA) and negative relative accommodation (NRA) were measured under bilateral viewing conditions with vision correction using a phoropter (target distance of $33 \mathrm{~cm}$ ). For PRA measurement, increasingly minus lenses were introduced in steps of $0.25 \mathrm{D}$, and the PRA endpoint was taken when the participant reports first blur. For NRA measurement, increasingly positive lenses were introduced in steps of $0.25 \mathrm{D}$, and the NRA endpoint was taken when the participant reports first blur. A minimum of two repeated measurements were performed.

\section{Questionnaire}

Questionnaires were completed by parents of the schoolchildren. Information on demographics (age and gender), lifestyle (time spent outdoors and time spent on near work activities) and other factors (parental myopia) were collected. The questionnaire asked for the average number of hours the child spent on performing outdoor activities per day, and on near work activities (such as reading and writing) per day.

\section{Statistical analyses}

The right eye was selected for analyses, as there were no significant differences in refractive error between right and left eyes at baseline $(-0.12 /-0.10 \times 61$ (SE of $-0.17 \pm 0.85 \mathrm{D}$ ) vs $-0.12 /-0.08 \times 71$ ( $\mathrm{SE}$ of $-0.17 \pm 0.91$ 
$\mathrm{D})$, respectively; Pearson correlation $=0.87$ ) and 2-year follow-up $(-0.56 /-0.13 \times 58$ (SE of $-0.63 \pm 1.39 \mathrm{D})$ vs $-0.49 /-$ $0.15 \times 50$ (SE of $-0.57 \pm 1.44 \mathrm{D})$, respectively; Pearson correlation $=0.92)$. Baseline characteristics of participants included and excluded were compared using the $\chi^{2}$ test or t-test. Incidence of myopia was defined as the onset of myopia during the 2-year follow-up period in participants without myopia at baseline examination. The changes in refraction and ocular biometry measurements over the 2-year follow-up period were calculated by subtracting the measurements taken at baseline (2014) from the measurements taken at 2-year follow-up (2016). Univariable associations between factors and myopia were tested using logistic regression. Associations of factors with myopia onset were assessed using multivariable-adjusted logistic regression models including covariables selected using stepwise backward methods. To test the predictive ability of the factors associated with myopia onset over a 2-year period, receiver operating characteristic (ROC) curves were plotted and the area under the curve (AUC) values were calculated. Coefficients of each predictor derived using the multivariable logistic regression models were generated, as shown in the equation:

$$
\ln \left(\frac{\mathrm{p}}{1-\mathrm{p}}\right)=\beta_{0}+\beta_{1} \mathrm{X}_{1}+\beta_{2} \mathrm{X}_{2}+\beta_{3} \mathrm{X}_{3}+\ldots+\beta_{\mathrm{n}} \mathrm{X}_{\mathrm{n}}
$$

where $\mathrm{p}$ is the probability of the participant becoming myopic over a 2-year period, $\beta_{\mathrm{i}}$ is the coefficient of factor $\mathrm{i}$, and $\mathrm{X}_{\mathrm{i}}$ is the value of factor $\mathrm{i}$. Analyses were performed using Python V.3.6.6. ${ }^{33-36}$

\section{RESULTS}

A total of 1022 (95.9\%) in 1066 participants attended the 2-year follow-up and had complete data at both baseline and 2-year follow-up. The mean age was $7.8 \pm 0.7$ (SD) years and $55.3 \%$ were male children. Of which, 762 and 260 children were from the urban and rural schools, respectively. There were no significant differences between included and excluded participants, except for a higher proportion from the rural school who were excluded $(\mathrm{p}<0.001$; table 1). Of 1022 schoolchildren from both schools, 192 (18.8\%; 95\% CI, 16.3\%-21.6\%) had myopia at baseline. The baseline prevalence of myopia was not significantly different between children from the urban school $(19.6 \%$; $95 \%$ CI, $16.6 \%$ to $22.9 \%)$ and the rural school (16.5\%; 95\% CI, $12.1 \%$ to $22.0 \%$; $\mathrm{p}=0.42$ ).

Over the 2-year period, the mean change in refractive error and AL among 1022 schoolchildren was $-0.44 /-0.03 \times 46$ ( $\mathrm{SE}$ of $-0.47 \pm 0.95 \mathrm{D})$ and $0.64 \pm 0.33 \mathrm{~mm}$, respectively. The mean 2-year change in refractive error and AL was $-1.07 /-0.17 \times 42$ (SE of $-1.45 \pm 0.88 \mathrm{D}$ ) and $0.94 \pm 0.33 \mathrm{~mm}$ for children who were already myopic at baseline $(\mathrm{N}=192),-1.22 /-0.06 \times 42$ (SE of $-1.25 \pm 0.73 \mathrm{D}$ ) and $0.93 \pm 0.27 \mathrm{~mm}$ for those who had myopia onset $(\mathrm{N}=229)$, and $0.07 /-0.02 \times-62$ (SE of $-0.05 \pm 0.42 \mathrm{D})$ and $0.44 \pm 0.19 \mathrm{~mm}$ for those who remained non-myopic ( $\mathrm{N}=601)$, respectively.

Of 830 children who were not myopic at baseline, the 2-year incidence of myopia was $27.6 \%$; 95\% CI, $24.2 \%$ to $31.3 \%$ ). The 2-year incidence of myopia was

\begin{tabular}{|c|c|c|c|}
\hline & $\begin{array}{l}\text { Included } \\
(\mathrm{N}=1022)\end{array}$ & $\begin{array}{l}\text { Excluded } \\
(\mathrm{N}=44)\end{array}$ & $P$ value \\
\hline Age, year & $7.8(0.7)$ & $7.9(0.7)$ & 0.25 \\
\hline \multicolumn{4}{|l|}{ Gender } \\
\hline Male & $565(55.3)$ & $28(63.6)$ & 0.35 \\
\hline Female & $457(44.7)$ & $16(36.4)$ & \\
\hline \multicolumn{4}{|l|}{ School } \\
\hline Urban & $762(74.6)$ & $19(43.2)$ & $<0.001$ \\
\hline Rural & $260(25.4)$ & $25(56.8)$ & \\
\hline \multicolumn{4}{|l|}{ Grade } \\
\hline 2 & $535(52.3)$ & $21(47.7)$ & 0.66 \\
\hline 3 & $487(47.7)$ & $23(52.3)$ & \\
\hline \multicolumn{4}{|l|}{ Number of myopic parents } \\
\hline 0 & $380(37.2)$ & $23(52.3)$ & 0.05 \\
\hline 1 & 387 (37.9) & $9(20.5)$ & \\
\hline 2 & $255(25.0)$ & $12(27.3)$ & \\
\hline Baseline spherical equivalent, D & $-0.2(0.8)$ & $-0.4(1.0)$ & 0.12 \\
\hline Baseline axial length, $\mathrm{mm}$ & $23.1(0.8)$ & $23.3(0.9)$ & 0.25 \\
\hline Presenting visual acuity, decimal & $1.1(0.4)$ & $1.0(0.4)$ & 0.26 \\
\hline $\begin{array}{l}\text { Negative relative accommodation, } \\
\text { D }\end{array}$ & $2.7(0.8)$ & $2.8(0.7)$ & 0.66 \\
\hline $\begin{array}{l}\text { Positive relative accommodation, } \\
\text { D }\end{array}$ & $-3.6(2.0)$ & $-3.1(1.8)$ & 0.05 \\
\hline Near work time, hours/day & $2.4(2.3)$ & $2.4(2.1)$ & 0.91 \\
\hline Outdoor time, hours/day & $2.2(1.9)$ & $2.2(1.8)$ & 0.97 \\
\hline
\end{tabular}

Data are expressed in $\mathrm{N}(\%)$ or mean (SD).

$\mathrm{D}$, diopter.

not significantly different between urban $(27.7 \%$; $95 \%$ CI, $23.8 \%$ to $32.1 \%$ ) and rural schools $(27.2 \%$; $95 \%$ CI, $20.8 \%$ to $34.7 \%$; $\mathrm{p}=0.90$ ), and was not significantly different among those aged 7 years $(26.8 \%$; 95\% CI, $21.6 \%$ to $32.8 \%$ ), aged 8 years $(28.4 \%$; $95 \%$ CI, $23.3 \%$ to $34.1 \%)$, and aged 9 years $(27.4 \%$; $95 \%$ CI, $18.9 \%$ to $38.0 \% ; \mathrm{p}=0.90$ ) as well. However, the 2-year incidence of myopia was significantly higher in children who were studying in grade 3 (30.3\%; 95\% CI, $25.0 \%$ to $36.2 \%$ ) than in grade $2(25.4 \%$; $95 \%$ CI, $21.0 \%$ to $30.3 \%$; $\mathrm{p}<0.001)$. Children studying in grade 3 were significantly older $(8.3 \pm 0.5$ vs $7.3 \pm 0.5$ years; $\mathrm{p}<0.001)$, had more negative SE $(-0.2 \pm 0.9$ vs $-0.1 \pm 0.7 \mathrm{D} ; \mathrm{p}=0.002)$, longer $\mathrm{AL}$ ( $23.2 \pm 0.8$ vs $23.0 \pm 0.8 \mathrm{~mm} ; \mathrm{p}<0.001)$, lower magnitude of NRA ( $2.7 \pm 0.8$ vs $2.8 \pm 0.8 \mathrm{D} ; \mathrm{p}=0.009)$, lower magnitude of PRA $(-3.4 \pm 1.9$ vs $-3.9 \pm 2.1 \mathrm{D} ; \mathrm{p}<0.001)$ and spent less time outdoors ( $1.6 \pm 0.9$ vs $2.8 \pm 2.3$ hours/day; $\mathrm{p}<0.001)$, compared with those studying in grade 2 .

In the univariable analyses, the odds of myopia onset over a 2-year period were higher in schoolchildren with two myopic parents, less hyperopic baseline SE, longer baseline AL and lower magnitude of baseline PRA measurements (table 2). In the multivariable analyses, female gender (adjusted OR of 2.2; $\mathrm{p}<0.001$ ), less hyperopic SE (adjusted OR of 11.5 per 1 myopic $\mathrm{D}$; $\mathrm{p}<0.001$ ), longer AL (adjusted 
Table 2 Risk of myopia onset over a 2-year period among schoolchildren in Wenzhou without myopia at baseline ( $N=830$ at risk)

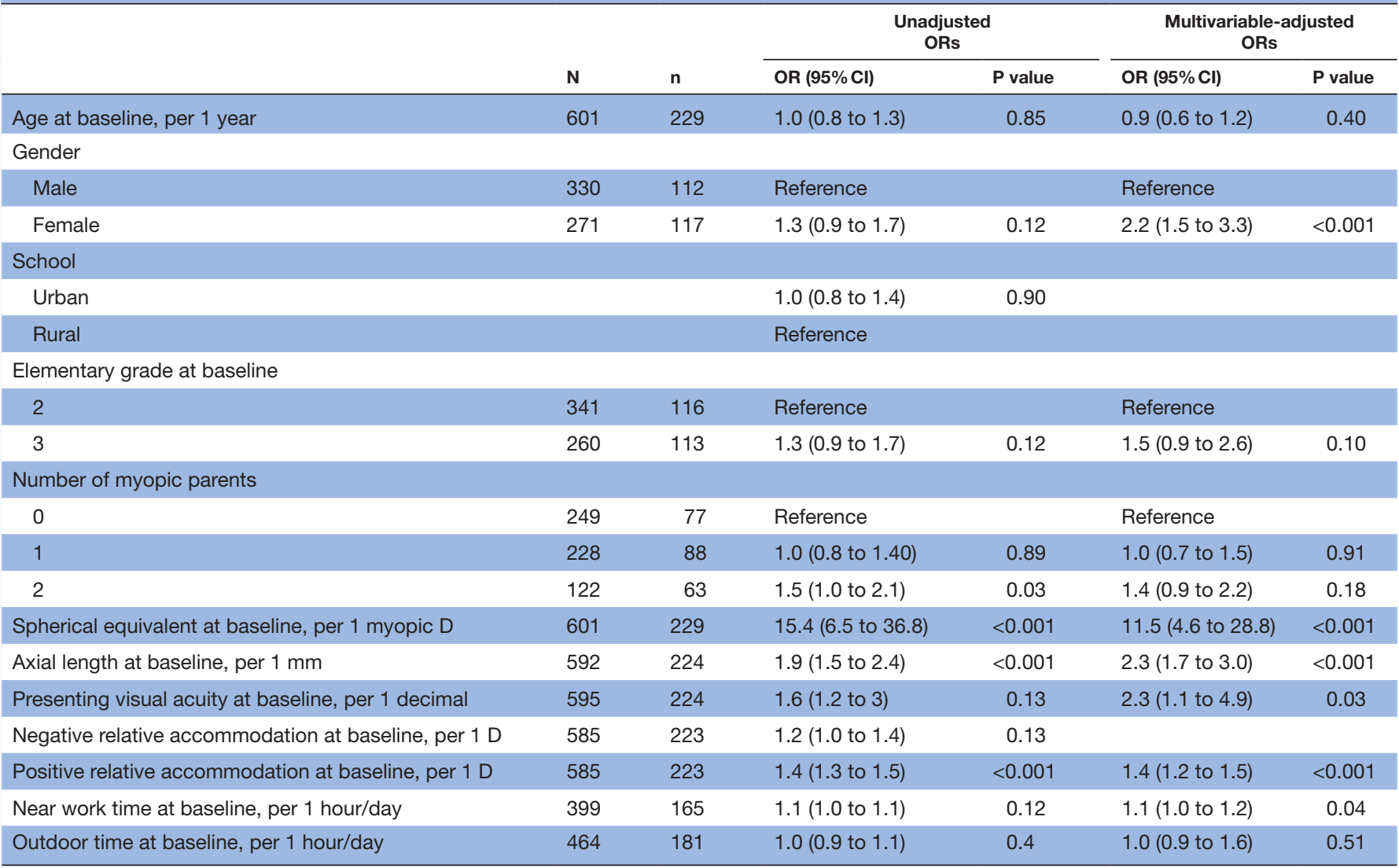

D, diopter.;

OR of 2.3 per $1 \mathrm{~mm}$ increase; $\mathrm{p}<0.001$ ), worse PVA (adjusted OR of 2.3 per 1 decimal; $\mathrm{p}=0.03$ ), longer time spent on near work (adjusted OR of 1.1 per 1 hour/day; $\mathrm{p}=0.04$ ) and lower magnitude of PRA (adjusted OR of 1.4 per $1 \mathrm{D}$ increase; $\mathrm{p}<0.001)$ measurements at baseline remained significantly associated with myopia onset.

The AUC of predictors of myopia onset was assessed in logistic regression analysis (table 3). Of these single predictors of myopia onset, PRA, SE and AL had the

\begin{tabular}{|c|c|c|}
\hline Factor & AUC & $95 \% \mathrm{Cl}$ \\
\hline Age & 0.47 & 0.41 to 0.52 \\
\hline Gender & 0.53 & 0.45 to 0.61 \\
\hline Elementary grade & 0.53 & 0.45 to 0.61 \\
\hline Parental myopia & 0.55 & 0.47 to 0.64 \\
\hline Spherical equivalent & 0.64 & 0.57 to 0.71 \\
\hline Axial length & 0.62 & 0.54 to 0.71 \\
\hline Presenting visual acuity & 0.53 & 0.44 to 0.61 \\
\hline Negative relative accommodation & 0.53 & 0.45 to 0.62 \\
\hline Positive relative accommodation & 0.66 & 0.58 to 0.74 \\
\hline Near work time & 0.55 & 0.45 to 0.65 \\
\hline Outdoor time & 0.53 & 0.38 to 0.62 \\
\hline
\end{tabular}

highest AUC values of 0.66 (95\% CI, 0.58 to 0.74 ), 0.64 (95\% CI, 0.57 to 0.71$)$, and 0.62 (95\% CI, 0.54 to 0.71 ), respectively. Figure 1 shows the ROC curves of several combinations of predictors using logistic regression models. The combination of age, gender, parental myopia and SE had an AUC of 0.65 (95\% CI, 0.57 to 0.72), which was not significantly higher than using SE alone (AUC of $0.64)$. The combination of age, gender, parental myopia, SE and AL had an AUC of 0.70 (95\% CI, 0.63 to 0.78), and combination of age, gender, parental myopia, SE, AL and PRA had an AUC of 0.74 (95\% CI, 0.68 to 0.80$)$. Addition of environmental factors, such as near work and outdoor time (combination of age, gender, parental myopia, SE, AL, PRA, near work time and outdoor time), did not significantly change the AUC.

\section{DISCUSSION}

Our study found a myopia incidence of $27.6 \%$ among schoolchildren aged 6-10 years in Wenzhou over a 2-year period. Female gender, less hyperopic SE, longer AL and lower magnitude of PRA were significant predictors of myopia onset. Of these single predictors of myopia onset, PRA, SE and AL alone had the highest predictive performance for myopia onset with AUC values of $0.66,0.64$ and 0.62 , respectively. The combination of age, gender, parental myopia, SE, AL and PRA achieved one of the 


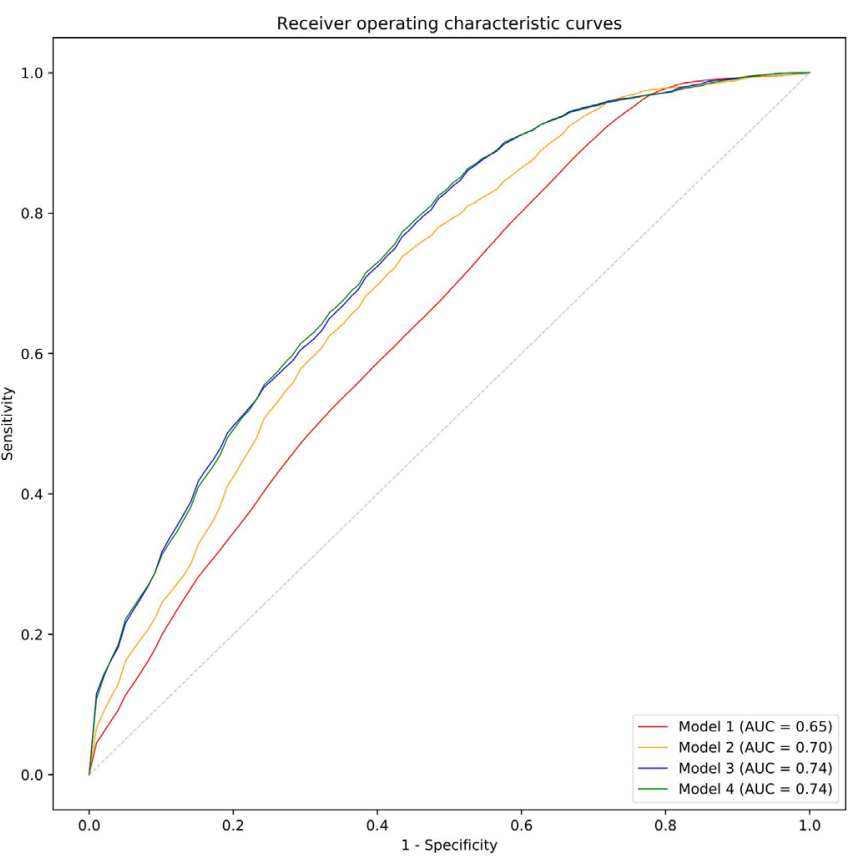

Figure 1 Receiver operating curves for prediction of myopia onset among schoolchildren in Wenzhou without myopia at baseline ( $\mathrm{N}=830$ at risk). Area under the curve (AUC) for model 1 based on combination of age, gender, parental myopia and spherical equivalent (SE) (red line) 0.65; AUC for model 2 based on combination of age, gender, parental myopia, SE and axial length (AL) (yellow line) 0.70; AUC for model 3 based on combination of age, gender, parental myopia, SE, AL and positive relative accommodation (PRA) (blue line) 0.74; AUC for model 4 based on combination of age, gender, parental myopia, SE, AL, PRA, near work time and outdoor time (green line) 0.74.

highest predictive performances (AUC of 0.74). The predictors elucidated may be useful in risk profiling of children who are predisposed to myopia onset for myopia prevention strategies in China.

\section{Incidence of myopia among children}

Several longitudinal studies in China have reported the incidence of myopia among schoolchildren with varying follow-up periods. ${ }^{8-101215}$ Compared with studies with follow-up periods of 2 years or more, the annualised incidence of myopia in our 2-year study in Wenzhou was $13.7 \%$, which was slightly lower than that of a 2-year study in Shanghai (18.1\%), ${ }^{11}$ but higher than that of a 5-year study in Chong Qing (10.6\%) and the 2.5-year study in Shunyi (7.8\%). ${ }^{915}$ Compared with studies with a 1-year follow-up period, the annualised incidence of myopia in our study was lower than the 1-year incidence of myopia among children aged 7-9 years in Guangzhou $(23.7 \% \text { to } 25.7 \%)^{12}$ and that of children aged $6-7$ years in rural Mojiang $(33.6 \%) .^{8}$ Direct comparison of findings is cautioned, as cycloplegia use, recruitment strategies, methodologies, follow-up period and age range of participants vary among the studies. The differences observed could also be due to the myopigenic environment (competitive education system and longer durations of near work activity) associated with the urban setting of Shanghai, compared with Wenzhou that comprises of both urban and rural settings, and rural settings in Chong Qing and Shunyi.

Compared with other East Asian populations, the annualised incidence of myopia in our study is similar to that among schoolchildren in Hong Kong (14.4\%) and Singapore (14.2\%). ${ }^{1037}$ However, a study in Taiwan reported a myopia incidence of $31.7 \%$, which was twofold higher than our findings. ${ }^{13}$ This may be explained by the increasing near work demands of children under a competitive educational system, or near proximity with the use of digital devices in the current modern environment. In contrast, children in Western countries have lower incidence of myopia than children in Asian countries, with annualised incidence of myopia among schoolchildren aged 5-11 years ranging from $2.2 \%$ in Northern Ireland, 2.4\% in Australia, to $2.8 \%$ in the USA, which is likely due to ethnic differences. ${ }^{16-18} 2027$

\section{Predictive models for myopia onset}

Numerous studies have built predictive models for myopia onset, with most based on cycloplegic refractive error measurements, 111618252728 and few based on non-cycloplegic ones. ${ }^{12}{ }^{20}$ Notably, the study conducted without cycloplegia by Wang et al in Guangzhou did not assess the predictive performance of factors associated with myopia onset. $^{12}$ In our study, the predictive performance of using non-cycloplegic refractive error alone for myopia onset was assessed and the AUC was 0.64, which was lower compared with the use of baseline cycloplegic refractive error as a single predictor of myopia onset in previous studies, with AUC ranging from 0.71 in a study in Xiamen to 0.88 in the Orinda Longitudinal Study of Myopia (OLSM) in the USA. ${ }^{11} 1618252728$ However, with addition of sociodemographic (age, gender, parental myopia), ocular biometry (AL) and accommodationrelated factors (PRA), a higher AUC of 0.74 was reached in our study. Similarly, the study in Xiamen showed that the use of baseline cycloplegic SE in combination with several factors, including gender, height, uncorrected VA and ocular biometry parameters, had higher predictive value in myopia onset prediction $(\mathrm{AUC}=0.97)$ than the use of baseline cycloplegic SE alone $(\mathrm{AUC}=0.71){ }^{25}$ This highlights the need for a combination of supplementary inputs for prediction of myopia onset. However, the added complexity in data collection needs to be acknowledged.

We found PRA as one of the strongest single predictors of myopia onset $(\mathrm{AUC}=0.66)$. A significant association between a lower magnitude of PRA and higher risk of myopia onset was found in our study. Although this coincides with findings of previous studies that were reported in the $1990 \mathrm{~s},{ }^{21-24}$ the lack of recent reports in the past decade indicates a need for future studies to corroborate the association between PRA and myopia onset. The magnitude of PRA was lower in premyopes than in non-myopes, suggesting 
the presence of reduced accommodative functions that may be associated with difficulty in increasing blurdriven accommodation in premyopes. ${ }^{22}{ }^{23} \mathrm{~A}$ reduced ability to accommodate at close distance for prolonged periods of near work may be associated with myopia onset. ${ }^{22} 23$ Therefore, accommodation and convergence function that are measured by common clinical tests can be used as a predictor for myopia onset.

Although non-cycloplegic SE was employed in our study, our findings showed baseline refractive error as one of the stronger single predictors of myopia onset, which was consistent with previous findings from the OLSM, the Collaborative Longitudinal Evaluation of Ethnicity and Refractive Error Study, the Sydney Adolescent Vascular and Eye Study and the study in Baoshan, China. ${ }^{11161827283839}$ In these studies, the performance of baseline cycloplegic refraction as a predictor of myopia onset surpassed that of ocular biometry parameters and environmental predictors. ${ }^{11} 16182728$ The consistent performance of SE in predicting myopia onset suggests that optical changes within the eye may exert greater influence on myopia onset, independent of sociodemographic factors (such as age, gender and parental myopia). Consistent with previous studies, ${ }^{812} 1318$ children with the hyperopic SE buffer had a significantly lower risk of myopia onset than those without.

Baseline AL was one of the stronger single predictors of myopia onset (AUC=0.64), which had slightly lower predictive performance than baseline refractive error. This phenomenon was also observed in previous studies. ${ }^{11} 1618272839$ The lower predictive performance of AL may be due to its larger variability, as it can be affected by other factors, such as age, gender and growth, ${ }^{40} 41$ and not be attributed to SE alone. Therefore, AL and noncycloplegic SE are complementary as relevant predictors of myopia onset.

Consistent with previous studies, ${ }^{9} 1012152541$ we found that female gender was associated with risk of myopia onset after multivariable adjustment. However, there are studies with non-significant gender-related association. ${ }^{8} 11$ 14 16-18 20 Similar to previous studies, our study found that the risk of myopia onset was not affected by age, ${ }^{811} 12161820$ parental myopia ${ }^{81520}$ and outdoor time. ${ }^{81142}$ The lack of significance for the age association may be due to the narrow age range in our study. Although some studies have showed an additional impact of environmental predictors on myopia onset, ${ }^{161826}$ our study found no association between outdoor time and myopia onset and borderline association between near work time and myopia onset, which may be due to the low variance of time spent outdoors and on near work among Chinese schoolchildren who are likely to have similar lifestyle patterns. ${ }^{811}$ The low variance in these environmental factors may also explain why the predictive performance of the combined model did not increase further.

\section{Strengths and limitations}

The strengths of our study include the longitudinal design of the cohort study with a 2-year follow-up period, high follow-up rate $(>95 \%)$ and standardised methodologies between visits. There are several limitations. First, the absence of cycloplegic refractive data may result in overestimation of myopic power and incidence of myopia. ${ }^{43}$ However, the evaluation of the predictive performance of non-cycloplegic SE for myopia onset remains crucial in a pragmatic setting. Second, the participants who were lost to follow-up were more likely to study in the rural school, compared with those who participated in the follow-up examinations. However, studying in either urban or rural schools was not associated with myopia onset in this study, thus the incidence of myopia is unlikely to be biased. Third, as the two schools in our study were not randomly selected, our study population may not be fully representative of the Chinese children population in Wenzhou (selection bias). The logistic regression model with a L2-regularisation was performed to check and prevent overfitting of data and to avoid the influence of extreme conditions. The predictive performance of the models (best AUC of 0.74) may need to be improved (for instance with new predictors) before further application. Further studies are needed to elucidate new predictors that may complement the use of non-cycloplegic methods in predicting myopia onset. Also, the current model is limited by the relatively narrow age range, but data from the 3-year follow-up visit will widen the age range once full data become available. Considering the variations in environmental exposures and cultures in different regions of China, generalisability of findings may be further limited. As the refraction was determined without cycloplegia, it may have an effect on the PRA and NRA values. However, subjective refraction was performed with fogging lenses that reduces the overestimation of myopic power. Lastly, the predictors were self-reported using questionnaire. Recall bias may be present, as participants have the tendency to overestimate the time spent outdoors and underestimate the time spent indoors. ${ }^{44} 45$ Therefore, the estimation of association between the predictors and myopia onset may be biased towards the null. However, the approach of using questionnaires is cost-effective and feasible in large cohort studies.

\section{Conclusion}

In conclusion, approximately one in four Chinese schoolchildren in Wenzhou had myopia onset over a 2-year period. The predictors of myopia onset include lower magnitude of PRA, less hyperopic SE, longer AL and female gender. This report provides an evaluation of the predictors of myopia onset for key stakeholders (parents, optometrists, clinicians and professionals at the primary eye-care level and clinical practice). Individuals at higher risk of myopia onset could be considered for closer monitoring, or targeted interventions that prevent or delay myopia onset. 
Contributors YY, BD, HC and JB conceived and designed the study. YY, YD, YYY and JB collected the data. YLW, YY, ST, BD and JB analysed and interpreted the data. YLW and YY wrote the manuscript. All authors reviewed and approved the final manuscript.

Funding This work was supported by the International S\&T Cooperation Programme of China (grant no. 2014DFA30940) and the Wenzhou Medical University-Essilor International Research Centre (WEIRC, grant nos. 95013006, 95016010).

Competing interests YLW, ST, DP and BD are employees of Essilor International, Singapore.

Patient and public involvement Patients and/or the public were not involved in the design, or conduct, or reporting, or dissemination plans of this research.

Patient consent for publication Not required.

Ethics approval Approval from the Ethics Committee of the Eye Hospital of Wenzhou Medical University was obtained (KYK[2013]34).

Provenance and peer review Not commissioned; externally peer reviewed.

Data availability statement Data are available upon request.

Open access This is an open access article distributed in accordance with the Creative Commons Attribution Non Commercial (CC BY-NC 4.0) license, which permits others to distribute, remix, adapt, build upon this work non-commercially, and license their derivative works on different terms, provided the original work is properly cited, appropriate credit is given, any changes made indicated, and the use is non-commercial. See: http://creativecommons.org/licenses/by-nc/4.0/.

\section{ORCID iDs}

Yee Ling Wong http://orcid.org/0000-0002-3331-7368

Binbin Su http://orcid.org/0000-0002-2269-3026

Yang Ding http://orcid.org/0000-0002-5102-1157

Hao Chen http://orcid.org/0000-0002-8594-5630

\section{REFERENCES}

1 Holden BA, Fricke TR, Wilson DA, et al. Global prevalence of myopia and high myopia and temporal trends from 2000 through 2050. Ophthalmology 2016;123:1036-42.

2 Wong Y-L, Saw S-M. Epidemiology of pathologic myopia in Asia and worldwide. Asia Pac J Ophthalmol 2016:5:394-402.

3 Chua SYL, Sabanayagam C, Cheung Y-B, et al. Age of onset of myopia predicts risk of high myopia in later childhood in myopic Singapore children. Ophthalmic Physiol Opt 2016;36:388-94.

4 Saw S-M, Gazzard G, Shih-Yen EC, et al. Myopia and associated pathological complications. Ophthalmic Physiol Opt 2005;25:381-91.

5 Saw S-M. How blinding is pathological myopia? Br J Ophthalmol 2006;90:525-6.

6 Pan C-W, Dirani M, Cheng C-Y, et al. The age-specific prevalence of myopia in Asia: a meta-analysis. Optom Vis Sci 2015;92:258-66.

7 Dong L, Kang YK, Li Y, et al. Prevalence and time trends of myopia in children and adolescents in China: a systemic review and metaanalysis. Retina 2020;40:399-411.

8 Li L, Zhong H, Li J, et al. Incidence of myopia and biometric characteristics of premyopic eyes among Chinese children and adolescents. BMC Ophthalmol 2018;18:178.

9 Zhao J, Mao J, Luo R, et al. The progression of refractive error in school-age children: Shunyi district, China. Am J Ophthalmol 2002;134:735-43

10 Fan DSP, Lam DSC, Lam RF, et al. Prevalence, incidence, and progression of myopia of school children in Hong Kong. Invest Ophthalmol Vis Sci 2004;45:1071-5.

$11 \mathrm{Ma} \mathrm{Y,} \mathrm{Zou} \mathrm{H,} \mathrm{Lin} \mathrm{S,} \mathrm{et} \mathrm{al.} \mathrm{Cohort} \mathrm{study} \mathrm{with} \mathrm{4-year} \mathrm{follow-up} \mathrm{of}$ myopia and refractive parameters in primary schoolchildren in Baoshan district, Shanghai. Clin Exp Ophthalmol 2018;46:861-72.

12 Wang SK, Guo Y, Liao C, et al. Incidence of and factors associated with myopia and high myopia in Chinese children, based on refraction without cycloplegia. JAMA Ophthalmol 2018;136:1017.

13 Tsai D-C, Fang S-Y, Huang N, et al. Myopia development among young schoolchildren: the myopia investigation study in Taipei. Invest Ophthalmol Vis Sci 2016;57:6852.

$14 \mathrm{Ku}$ P-W, Steptoe A, Lai Y-J, et al. The associations between near visual activity and incident myopia in children: a nationwide 4-year follow-up study. Ophthalmology 2019;126:214-220.

15 Zhou W-J, Zhang Y-Y, Li H, et al. Five-Year progression of refractive errors and incidence of myopia in school-aged children in Western China. J Epidemiol 2016;26:386-95.
16 French AN, Morgan IG, Mitchell P, et al. Risk factors for incident myopia in Australian schoolchildren: the Sydney adolescent vascular and eye study. Ophthalmology 2013;120:2100-8

17 McCullough SJ, O'Donoghue L, Saunders KJ. Six year refractive change among white children and young adults: evidence for significant increase in myopia among white UK children. PLoS One 2016;11:e0146332.

18 Jones LA, Sinnott LT, Mutti DO, et al. Parental history of myopia, sports and outdoor activities, and future myopia. Invest Ophthalmol Vis Sci 2007:48:3524.

19 Saxena R, Vashist P, Tandon R, et al. Incidence and progression of myopia and associated factors in urban school children in Delhi: the North India myopia study (nim study). PLoS One 2017;12:e0189774.

20 Guggenheim JA, Northstone K, McMahon G, et al. Time outdoors and physical activity as predictors of incident myopia in childhood: a prospective cohort study. Invest Ophthalmol Vis Sci 2012;53:2856.

21 Drobe B, de Saint-André R. The pre-myopic syndrome. Ophthalmic Physiol Opt 1995;15:375-8.

22 Gwiazda J, Thorn F, Bauer J, et al. Myopic children show insufficient accommodative response to blur. Invest Ophthalmol Vis Sci 1993;34:690-4

23 Goss DA. Clinical accommodation and heterophoria findings preceding juvenile onset of myopia. Optom Vis Sci 1991;68:110-6.

24 Goss DA, Jackson TW. Clinical findings before the onset of myopia in youth: 3. Heterophoria. Optom Vis Sci 1996;73:269-78.

25 Zhang M, Gazzard G, Fu Z, et al. Validating the accuracy of a model to predict the onset of myopia in children. Investig Ophthalmol Vis Sci 2011;52:5836-41.

26 Tideman JWL, Polling JR, Jaddoe VWV, et al. Environmental risk factors can reduce axial length elongation and myopia incidence in 6- to 9-year-old children. Ophthalmology 2019;126:127-36.

27 Zadnik K, Sinnott LT, Cotter SA, et al. Prediction of juvenile-onset myopia. JAMA Ophthalmol 2015;133:683.

28 Zadnik K, Mutti DO, Friedman NE, et al. Ocular predictors of the onset of juvenile myopia. Invest Ophthalmol Vis Sci 1999;40:193643.

29 Wakayama A, Nishina S, Miki A, et al. Incidence of side effects of topical atropine sulfate and cyclopentolate hydrochloride for cycloplegia in Japanese children: a multicenter study. Jpn J Ophthalmol 2018;62:531-6.

30 Wildsoet CF, Chia A, Cho P. Imi - interventions myopia Institute: interventions for controlling myopia onset and progression report. Investig Ophthalmol Vis Sci 2019.

31 Kaye SB. Objective evaluation of refractive data and astigmatism: quantification and analysis. Eye 2014;28:154-61.

32 Flitcroft $\mathrm{DI}, \mathrm{He} \mathrm{M}$, Jonas JB, et al. Imi - defining and classifying myopia: a proposed set of standards for clinical and epidemiologic studies. Investig Ophthalmol Vis Sci 2019;60:M20-30.

33 Oliphant T, Millma JK. A quide to NumPy. Trelgol Publ, 2006.

34 McKinney W. Data structures for statistical computing in python. Proc 9th Python Sci Conf 2010.

35 Pedregosa F, Varoquaux G, Gramfort A. Scikit-learn: machine learning in python. J Mach Learn Res 2011;12:2825-30.

36 Seabold S, Perktold J. Econometric and statistical modeling with python. Proc 9th Python Sci Conf 2010

37 Saw S-M, Tong L, Chua W-H, et al. Incidence and progression of myopia in Singaporean school children. Invest Ophthalmol Vis Sci 2005;46:51.

38 Jones-Jordan LA, Sinnott LT, Manny RE, et al. Early childhood refractive error and parental history of myopia as predictors of myopia. Invest Ophthalmol Vis Sci 2010;51:115.

39 Mutti DO, Hayes JR, Mitchell GL, et al. Refractive error, axial length, and relative peripheral refractive error before and after the onset of myopia. Invest Ophthalmol Vis Sci 2007;48:2510.

40 Wang D, Ding X, Liu B, et al. Longitudinal changes of axial length and height are associated and concomitant in children. Invest Ophthalmol Vis Sci 2011;52:7949.

41 Saw SM, Chua WH, Gazzard G, et al. Eye growth changes in myopic children in Singapore. Br J Ophthalmol 2005;89:1489-94.

42 Saw S-M, Shankar A, Tan S-B, et al. A cohort study of incident myopia in Singaporean children. Invest Ophthalmol Vis Sci 2006; $47: 1839$.

43 Hashemi H, Khabazkhoob M, Asharlous A, et al. Cycloplegic autorefraction versus subjective refraction: the Tehran eye study. $\mathrm{Br}$ J Ophthalmol 2016;100:1122-7.

44 Jones-Jordan LA, Sinnott LT, Cotter SA, et al. Time outdoors, visual activity, and myopia progression in juvenile-onset myopes. Invest Ophthalmol Vis Sci 2012:53:7169.

45 Alvarez AA, Wildsoet CF. Quantifying light exposure patterns in young adult students. J Mod Opt 2013;60:1200-8. 